lows from our lemma by much the same sort of argument that produced our basic theorem.

\title{
REFERENCES
}

1. E. A. Coddington and N. Levinson, Theory of ordinary differential equations, McGraw-Hill, New York, 1955.

2. L. M. Graves, The theory of functions of real variables, 2nd ed., McGraw-Hill, New York, 1956.

3. J. LaSalle, Uniqueness theorems and successive approximations, Ann. of Math. 50 (1949), 722-730.

Harpur College

\section{UNCOUNTABLY MANY NONISOMORPHIC NILPOTENT LIE ALGEBRAS ${ }^{1}$}

\section{CHONG-YUN CHAO}

Throughout this note, $L$ denotes a Lie algebra over the real number field $R$. We shall define $L^{i}$ and $L_{i}$ inductively. $L=L^{0}=L_{0}, L^{i}$ $=\left[L^{i-1}, L^{i-1}\right]$, and $L_{i}=\left[L, L_{i-1}\right]$ for all integers $i \geqq 1$. Thus, $L^{i}$ is the space of all finite sums $\sum[x, y], x, y \in L^{i-1}$. Similarly, $L_{i}$ is the space of all finite sums $\sum[x, y], x \in L$ and $y \in L_{i-1}$. If $L^{r}=0$ and $L^{r-1} \neq 0$, $L$ is said to be solvable of index $r$. If $L_{t}=0$ and $L_{t-1} \neq 0, L$ is said to be nilpotent of length $t$.

Definition. Let $F$ be a subfield of $R$. A Lie algebra $L$ over $R$ is said to be an $F$-algebra if its structure constants with respect to some basis of $L$ lie in $F$.

Malcev [1] showed that for each integer $n \geqq 16$ there is a nilpotent Lie algebra of length 2 and dimension $n$ which is not a rational algebra. The purpose of this note is to prove the following theorem which contains an improvement of Malcev's result:

THEOREM. There exist uncountably many nonisomorphic nilpotent Lie algebras of length 2 for any given dimension $N \geqq 10$.

Following from the theorem we can easily show:

Corollary 1. There exist uncountably many solvable not nilpotent Lie algebras of index 3 for any given dimension $M \geqq 11$.

Received by the editors November 13, 1961.

1 This is a portion of my thesis submitted to the University of Michigan. I am deeply grateful to Professor H. Samelson for his guidance and assistance. This work was supported by the contract AF 49(648)-104 and Lotta B. Backus scholarship. 
Let $E$ be a subfield of $R$, let $m, n$ be two natural numbers, and let $c_{j k}^{i}, i=1,2, \cdots, n, j, k=1,2, \cdots, m$, be real numbers such that $c_{j k}^{i}=-c_{k j}^{i}$. Also let $L$ be a Lie algebra over $R$ defined by a basis $\left(x_{1}, \cdots, x_{m}, y_{1}, \cdots, y_{n}\right)$ with products $\left[x_{j}, x_{k}\right]=\sum_{i=1}^{n} c_{j k}^{i} y_{i}$ for $j, k=1,2, \cdots, m$, and all other products zero, so that $L$ is nilpotent of length $\leqq 2$.

LEMMA. If the numbers $c_{j k}^{i}, 1 \leqq i \leqq n, 1 \leqq j<k \leqq m$, are algebraically independent over $E$, and if $(n / 2)\left(m^{2}-m\right)>m^{2}+n^{2}$, then $L$ is not an E-algebra.

Proof. We first note that $(n / 2)\left(m^{2}-m\right)>m^{2}+n^{2}$ implies $(1 / 2)\left(m^{2}-m\right)>n$. Any $n$ different elements $\left[x_{j}, x_{k}\right], j<k$, of $L_{1}$ are linearly independent, since the determinant formed by $c_{j k}^{i}$ involved cannot be zero by the algebraic independence of all $c_{j k}^{i}$. It follows that $L_{1}$ is generated by $y_{1}, y_{2}, \cdots, y_{n}$, denoted by $L_{1}=\left(\left(y_{1}, y_{2}, \cdots, y_{n}\right)\right)$, since for any $x \in L_{1}$ there exist $u_{i}, v_{i}$ such that $x=\sum_{i}\left[u_{i}, v_{i}\right]$ which is a linear combination of $y_{i}$ 's. We also note that the center of $L$ is exactly $L_{1}$; let $x$ be any element of the center, then $x=\sum_{j=1}^{m} a_{j} x_{j}$ $+\sum_{r=1}^{n} b_{r} y_{r}$ and $0=\left[x, x_{k}\right]=\sum_{j=1}^{m} a_{j} \sum_{i=1}^{n} c_{j k}^{i} y_{i}$ for $k=1,2, \cdots, m$. By linear independence of the $\left\{y_{i}\right\}$, we have $\sum_{j=1}^{m} a_{j} c_{j k}^{i}=0$ for $i=1,2, \cdots, n$, and $k=1,2, \cdots, m$, i.e., there are $n \cdot m$ equations and $m$ unknowns. By the algebraic independence of all $c_{j k}^{i}$, the rank of the coefficient matrix in the system of homogeneous equations is equal to $m$. Hence, we have $a_{1}=a_{2}=\cdots=a_{m}=0$. Consequently, $x=\sum_{r=1}^{n} b_{r} y_{r}$ and the center is $L_{1}$, and $L$ is of length 2 .

Suppose now that $L$ is an $E$-algebra with basis $\left(z_{1}, \cdots, z_{m}, z_{m+1}, \cdots, z_{m+n}\right)$ and structure constants $d_{j k}^{i}, 1 \leqq i, j, k$ $\leqq m+n$, lying in $E$. We can assume that $\left(z_{1}, \cdots, z_{m}\right)$ are independent modulo $L_{1}$, i.e., they span a complement $C$ of $L_{1}$, in $L$. We can write $z_{m+i}=v_{i}+t_{i}$ with $v_{i} \in C$ and $t_{i} \in L_{1}$ for $i=1,2, \cdots, n$. Clearly, $\left(z_{1}, \cdots, z_{m}, t_{1}, \cdots, t_{n}\right)$ is still a basis for $L$. We have

$$
\left[z_{i}, z_{j}\right]=\sum_{r=1}^{m} d_{i j}^{r} z_{r}+\sum_{s=m+1}^{m+n} d_{i j v_{s-m}^{s}}^{s}+\sum_{s=m+1}^{m+n} d_{i j}^{s} t_{s-m},
$$

for $1 \leqq i, j \leqq m$. But since $\left[z_{i}, z_{j}\right] \in L_{1}$, the first two sums, which are in $C$, must be zero. Hence we have

$$
\left[z_{i}, z_{j}\right]=\sum_{r=1}^{n} d_{i j}^{m+r} t_{r}, \quad \text { for } \quad i, j=1,2, \cdots, m .
$$

These equations describe the multiplication in $L$ in the basis $\left(z_{1}, \cdots, z_{m}, t_{1}, \cdots, t_{n}\right)$; the structure constants are part of the structure constants for the basis $\left(z_{1}, \cdots, z_{m}, z_{m+1}, \cdots, z_{m+n}\right)$. 
We note that $\left(\left(x_{1}, \cdots, x_{m}\right)\right)$ also forms a complement of $L_{1}$ in $L$ say $C^{\prime}$. It follows that we can replace each $z_{i}$ by an element $s_{i}$ such that $s_{i}-z_{i} \in L_{1}$ and $s_{i} \in C^{\prime}$. Since $L_{1}$ is the center of $L$, the structure constants for the basis $\left(s_{1}, \cdots, s_{m}, t_{1}, \cdots, t_{n}\right)$ are the same as for the basis $\left(z_{1}, \cdots, z_{m}, t_{1}, \cdots, t_{n}\right)$ above.

The set of vectors $\left\{s_{1}, \cdots, s_{m}\right\}$ is of course a basis for $C^{\prime}$, and, therefore, we have $s_{i}=\sum_{p=1}^{m} a_{i p} x_{p}, i=1, \cdots, m$, where $A=\left(a_{i p}\right)$ is a nonsingular matrix. Similarly, $t_{g}=\sum_{r=1}^{n} b_{o r} y_{r}, g=1, \cdots, n$, with nonsingular matrix $B=\left(b_{o r}\right)$. Substituting into $\left[s_{i}, s_{j}\right]=\sum_{u=1}^{n} d_{i j}^{m+u} t_{u}$, $1 \leqq i, j \leqq m$, we obtain, by linear independence,

$$
\sum_{p} \sum_{o} a_{i p} a_{j g} c_{p g}^{r}=\sum_{u} d_{i j}^{m+u} b_{u r}
$$

for fixed $i, j$, and $r$.

This means, with $\bar{a}_{i j}=\left(A^{-1}\right)_{i j}$, that

$$
c_{p o}^{r}=\sum_{i} \sum_{j} \sum_{u} d_{i j}^{m+u} b_{u r} \bar{a}_{p i} \bar{a}_{o j}
$$

These equations imply that the $c_{p o}^{r}$ lie in the field $E\left(a_{i p}, b_{u r}\right)$, but this field has degree of transcendency over $E$ at most $m^{2}+n^{2}$ which is a contradiction. Hence, $L$ is not an $E$-algebra.

The smallest dimension to which this applies is 10 with $m=6$ and $n=4$. In fact, the lemma applies to any dimension $N \geqq 10$, because when $N \geqq 10, N^{2}-10 N+8>0$ holds, implying that $n\left(m^{2}-m\right) / 2$ $>m^{2}+n^{2}$ holds for $n=4$ and $m=N-4$.

Now the proof of the theorem: It is well known that there exists a set, $S$, of uncountably many real numbers which are algebraically independent over the rational number field $Q$. With $n=4$ and $m=N-4$, we divide $S$ into disjoint subsets $\left(c_{j k}^{i}\right)_{\alpha}$ (the Greek index distinguishes the various subsets), each of which is restricted to values of $j$ and $k$ such that $j<k$ and $c_{j k}^{i}=-c_{k j}^{i}$. Write $(L)_{\alpha}$ $=\left(\left(x_{1}, \cdots, x_{m}, y_{1}, \cdots, y_{4}\right)\right)$ with products $\left[x_{j}, x_{k}\right]=\sum_{i=1}^{4} c_{j k}^{i} y_{i}$, $j, k=1,2, \cdots, m$, and all other products zero. There are still uncountably many such subsets $\left\{c_{j k}^{i}\right\}$ since each $\left\{c_{j k}^{i}\right\}$ is finite. Consequently, there are uncountably many such Lie algebras $(L)_{\alpha}$. We claim that any two $(L)_{\alpha}$ and $(L)_{\alpha^{\prime}}$ are nonisomorphic. Since $\left(c_{j k}^{i}\right)_{\alpha}$ are algebraically independent over $Q\left(\left\{\left(c_{j k}^{i}\right)_{\alpha^{\prime}}\right\}\right)$, apply the lemma with $E=Q\left(\left\{\left(c_{j k}^{i}\right)_{\alpha^{\prime}}\right\}\right)$.

Now the proof of Corollary 1: In the proof of the theorem we have seen that for each $\alpha,(L)_{\alpha}=\left(\left(x_{1}, \cdots, x_{N-4}, y_{1}, \cdots, y_{4}\right)\right)$, with $\left[x_{j}, x_{k}\right]=\sum_{i=1}^{4} c_{j k}^{i} y_{i}$ for $j, k=1, \cdots, N-4$ where $N \geqq 10$, and all 
other products zero, is a nilpotent Lie algebra of dimension $N$ and length 2 . Let $\left(L^{\prime}\right)_{\alpha}=\left(\left(x_{1}, \cdots, x_{N-4}, y_{1}, \cdots, y_{4}, z_{\alpha}\right)\right)$ where the multiplications of $x_{j}^{\prime}$ 's and $y_{i}^{\prime}$ 's are defined as same as in $L$ and $\left[z_{\alpha}, x_{j}\right]=x_{j}$, $\left[z_{\alpha}, y_{i}\right]=2 y_{i}$ for $j=1, \cdots, N-4 ; i=1, \cdots, 4$ and $N \geqq 10$. Then clearly, $(L)_{\alpha}$ is a solvable not nilpotent Lie algebra of dimension $M=N+1 \geqq 11$. Any two such Lie algebra $\left(L^{\prime}\right)_{\alpha^{\prime}}$ and $\left(L^{\prime}\right)_{\alpha}$ are clearly nonisomorphic because by the theorem their commutators are nonisomorphic.

COROLlaRY 2. There are uncountably many nonisomorphic nonrational nilpotent Lie algebras of length 2 for any given dimension $N \geqq 10$.

REMARKS. We note that the uncountability of nonisomorphic solvable Lie algebras is quite different from the case of semisimple Lie algebras where in each dimension there are only a finite number of nonisomorphic ones.

\section{BIBLIOGRAPHY}

1. A. I. Malcev, On a class of homogeneous spaces, Izv. Akad. Nauk SSSR Ser. Mat. 13 (1949), 9-32; Amer. Math. Soc. Transl. No. 39 (1951).

Research Center, International Business Machines YORKTOWN HeIGHTS, NEW YORK 